\title{
Access to Justice: the Quality of the Procedure
}

\author{
Laura Klaming \\ Molengraaff Institute for Private Law, Faculty of Law, Utrecht University; \\ Hague Institute for the Internationalisation of Law (HIIL); Tilburg University, \\ Tilburg Institute for Interdisciplinary Studies of Civil Law \\ and Conflict Resolution Systems (TISCO); The Study Group Access to \\ Justice \\ L.Klaming@law.uu.nl
}

$\&$

Ivo Giesen

Molengraaff Institute for Private Law, Faculty of Law, Utrecht University; Ius Commune Research School; Hague Institute for the Internationalisation of Law (HIIL); The Study Group Access to Justice

I.Giesen@law.uu.nl

\section{TISCO Working Paper Series on Civil Law and Conflict Resolution Systems}

No. 002/2008

February, 2008, Version: 1.0

\footnotetext{
" This study was facilitated in part by the Hague Institute for the Internationalisation of Law (HiiL) through the sponsoring of a research project titled Measuring Access to Justice in a Globalising World, The Hague Model of Access to Justice.

** The Study Group Access to Justice consist of researchers with different backgrounds from Tilburg University and Utrecht University. The aim of the 'Measuring Access to Justice in a Globalising World. The Hague Model of Access to Justice' is to develop, operationalise and pilot test a methodological framework for measuring the costs and quality of access to justice in international and national settings. If you are interested in more information about the project, please email to: j.m.barendrecht@uvt.nl or visit our website: http://www.tilburguniversity.nl/faculties/law/research/cva/access/
} 
Abstract: The quality of procedures is believed to play an important role in ccess to justice. It is assumed, that the quality of a procedure is high if people evaluate it as fair. Fair procedures are likely to improve access to justice. For the purpose of the present paper, the quality of procedures is determined in terms of people's perceptions of procedural justice. Procedural justice refers to various aspects that a procedure should meet in order to be perceived as fair by its users. Research on procedural justice has a long tradition within the field of social psychology. It was therefore decided to focus on the theoretical and empirical framework of social psychology in order to define and evaluate the quality of procedures. Other frameworks, such as legal, philosophical and economic perspectives, are not discussed here. Various indicators of procedural justice as well as potential factors influencing people's evaluations of procedural justice have been identified in past research. Factors that were found to affect procedural justice perceptions include cultural aspects, features of the outcome, as well as characteristics of the dispute. In addition, recent research has indicated that people may also rely on emotional information when evaluating procedural justice. This aspect seems to be of particular importance in the legal context as conflicts are generally characterised by negative emotions which may then influence people's justice judgments. The present paper discusses the most relevant research findings and theories of procedural justice. In addition, the different conceptualisations of justice and their relevance for the purpose of evaluating the quality of procedures are discussed in more detail in order to determine which indicators of procedural justice are most relevant and should therefore be incorporated into a measurement method to assess the quality of procedures. 
Table of Contents

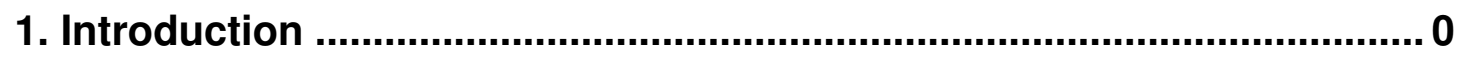

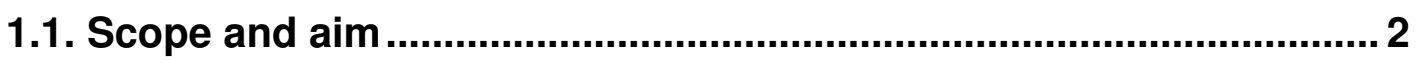

2. Procedural justice ................................................................................ 3

2.1. Indicators of procedural justice...................................................... 5

2.2. Why people value procedural justice ................................................ 9

2.2.1. Referent cognition theory ....................................................... 9

2.2.2. Relational models ................................................................... 10

2.2.3. Fairness heuristic theory ..................................................... 12

3. Conceptualisations of justice ........................................................ 14

4. Factors influencing justice judgments............................................ 20

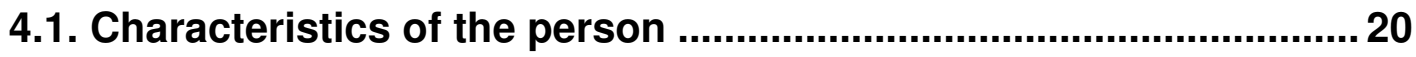

4.2. Culture ................................................................................................ 21

4.3. Outcome information and outcome favourability .......................... 22

4.4. Characteristics of the dispute.................................................... 23

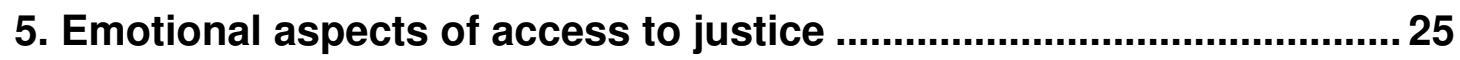

6. Conclusion and outline for future research.......................................... 29

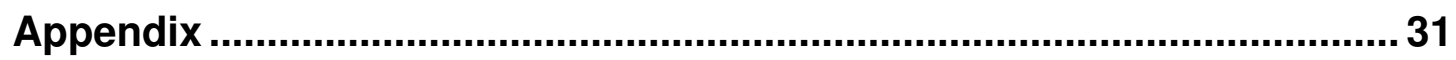

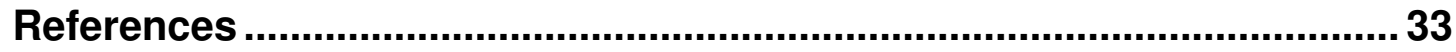




\section{Introduction}

Neither costs nor other barriers should prevent any individual or group from having access to justice whenever they are faced with a problem that needs resolution by a neutral person or neutral body, including the police, the courts or more informal dispute resolution services such as mediators or arbitrators. Most certainly, high monetary costs, time costs as well as limited knowledge of legal rules and procedures and the justice system in general pose barriers to access to justice. In addition to economic costs, people who need access to justice experience psychological costs that may function as a barrier to access to justice. These psychological costs can be defined in terms of perceptions of procedural justice.

Procedural justice refers to various aspects that a procedure should meet in order to be perceived as fair by its user. People's perceptions of procedural justice are also likely to be influenced by emotional distress both as a consequence of the conflict itself and the fact that one has to take legal action in order to have the conflict resolved. As legal procedures are unfamiliar to most people and the outcome is unknown prior to entering into a procedure, this is likely to cause feelings of distress and uncertainty. Consequently, people who embark on legal action most probably feel emotionally distressed both before entering into a legal procedure and during the procedure itself. Depending on the fairness of the procedure and its outcome people may also feel distressed after an outcome has been reached. It is therefore believed that affect ${ }^{1}$ is an important determinant of procedural justice perceptions. Evaluating procedural justice perceptions in the light of people's affective state has been suggested to be beneficial in order to foster a better understanding of the psychological mechanisms underlying people's justice evaluations (Van den Bos, 2003).

The psychological costs of a procedure are believed to determine (at least to a great extent) the quality of a legal procedure as perceived by its users. The quality of a legal procedure is determined here in terms of people's evaluations of procedural justice. It is of course acknowledged that other theoretical frameworks could also be used to define the quality of a

\footnotetext{
${ }^{1}$ In line with what other researchers have suggested, affect refers to both mood and emotions (Forgas, 2002; Van den Bos, 2003).
} 
procedure, such as philosophical and economic views of procedural goods or legal procedure rules. However, for the purpose of the present paper, insights from social psychology research are relied upon in order to determine the quality of a procedure. This approach has been chosen because the vast majority of research on how people experience justice comes from the field of social psychology ${ }^{2}$. It was therefore decided to focus on the theoretical and empirical framework of social psychology in order to define and evaluate the quality of procedures.

It is believed that the quality of a procedure will be high if an individual evaluates a procedure as fair. A procedure that has a high quality most certainly improves access to justice. Fair procedures were found to enhance many important cognitions, attitudes, feelings, and behaviours. Research has demonstrated that the benefits of procedural justice include an increased satisfaction with and acceptance of decisions and outcomes, and enhanced obedience to laws (Greenberg, 1987; Lind, Kulik, Ambrose, \& de Vera Park, 1993; Thibaut \& Walker, 1975; Tyler, 2006). It has furthermore been argued that the belief that one has been treated fairly by the authorities enhances perceptions of the legitimacy of those authorities and increases the transparency of procedures to the public (O'Hear, 2007). The positive effect of fair procedures has been termed 'fair process effect' (Folger, Rosenfield, Grove, \& Corkran, 1979).

Unfair procedures, in contrast, have negative consequences. Moreover, unfair events generally affect people's feelings and behaviours more strongly than fair events because bad information is processed more thoroughly than good information (Baumeister, Bratslavsky, Finkenauer, \& Vohs, 2001; Törnblom \& Vermunt, 1999). The finding that injustice probably has a greater influence on people's feelings and behaviour than justice has been explained in terms of people's expectations (Van den Bos, Vermunt, \& Wilke, 1996; Van den Bos \& Van Prooijen, 2001). While fair procedures confirm expectations,

\footnotetext{
${ }^{2}$ There is an excessive amount of research from social psychology on procedural justice. Economic and political views of procedural goods seem to rely on this body of research. Within the economic literature, it has only recently been recognised that people not only care about outcomes but also about the procedures that lead to those outcomes. It has been argued that procedural justice - or procedural utility as it has been termed within this specific context - is an important source of human well-being because it enhances feelings of selfdetermination (Frey, Benz, \& Stutzer, 2004; Frey \& Stutzer, 2005).
} 
unfair procedures strongly violate people's expectations of fairness. This means that if people who are involved in a legal dispute feel that they are being unfairly treated by the authority this has a greater impact on their subsequent reactions than when they feel that they are being fairly treated. In fact, procedural injustice was found to prompt protest behaviour, impede compliance with decisions and even cause recidivism (Van den Bos, 2005). Moreover, the belief that one has been treated unfairly has been shown to prompt the initiation of lawsuits (Lind, Greenberg, Scott, \& Welchans, 2000). In addition, within the organisational context unfair procedures were found to lead to resentment and retaliation (De Cremer, 2006; Folger, 1987).

\subsection{Scope and aim}

This paper discusses one aspect of a broader research project known as "Measuring Access to Justice in a Globalising World. The Hague Model of Access to Justice". The aim of this research project is to obtain a standard methodology for measuring the costs and quality which average users may expect on the most common paths to justice ${ }^{3}$.

In a situation in which people need access to justice, e.g. in order to protect their rights or obtain what they are entitled to, they have to choose one of many distinct paths to have their conflict resolved. These paths include negotiation, administrative procedures with a governmental agency as well as legal proceedings. People can choose between those distinct procedures taking the nature of the conflict and their needs into account. However, there is not yet a measure that assists people in choosing the right procedure. Additionally, both users and providers of justice currently have no instrument to evaluate the quality of distinct procedures. The aim of the "Measuring Access to Justice" research project is to develop an instrument that enables both individuals and providers of justice to compare distinct paths in terms of

\footnotetext{
${ }^{3}$ In previous stages of the "Measuring Access to Justice in a Globalising World. The Hague Model of Access to Justice" research project, the most urgent legal needs and associated paths to justice have been identified (M. Barendrecht, P. Kamminga, \& J.H. Verdonschot, Identifying legal needs: a bottom up approach to rule of law and access to justice, 2007). In addition, an approach to measuring the costs of access to justice has been determined (M. Barendrecht, J. Mulder, \& I. Giesen, How to measure the price and quality of access to justice?, 2006). If you are interested in more information about these papers or the research project, please email to: j.m.barendrecht@uvt.nl or visit our website: http://www.tilburguniversity.nl/faculties/law/research/cva/access/
} 
their costs and quality. More specifically, this standard methodology is to include (a) indications as to the costs of a procedure, (b) indications as to the quality of a procedure, and (c) indications as to the quality of the outcome of a procedure. These aims are achieved by (a) measuring the money and time people spend in order to obtain a resolution to the conflict as well as emotional costs associated with the procedure, (b) measuring people's perceptions of the fairness of the procedure used to obtain a resolution to the conflict, and (c) measuring people's perceptions of the fairness of the outcome.

The purpose of this paper is to describe the relevant insights of research on procedural fairness concerning project $b$. The aim of project $b$ "The quality of the procedure" is to obtain a method that makes the quality of the paths to justice transparent. Specifically, it aims at developing a measurement tool by means of which the quality of distinct paths to justice can be assessed. Since, for the purpose of the study, the quality of procedures is defined in terms of procedural justice, the relevant indicators of procedural justice need to be determined. These indicators are addressed in more detail in the following sections. An overview of the relevant literature and research findings of procedural justice is also given. There is an ongoing debate concerning the conceptualisation of justice. Because of its relevance for the purpose of the present paper, the discussion on the conceptualisation of justice is addressed in the light of recent empirical findings. Additionally, the potential influence of several factors on procedural justice perceptions, and most importantly the link between affect and procedural justice, and its relevance with regard to justice judgments in general and justice judgments within the legal context in particular are discussed in more detail. Finally, the implications of this literature review for the purpose of the present project and the proposed measure to evaluate the quality of procedures are outlined.

\section{Procedural justice}

The notion of justice has received a large amount of research interest over the past 30 years. Generally, people who are involved in a legal conflict are interested in receiving favourable outcomes. However, people are less 
concerned with the outcome than is commonly assumed (Lind \& Tyler, 1988; Thibaut \& Walker, 1975). The initial belief that people are primarily concerned with the outcomes they receive has been referred to as distributive justice (Rawls in Crombag \& Van Koppen, 2002; Thibaut \& Walker, 1975). Specifically, it has been argued that the outcome should reflect the effort people invest in something and that people judge an outcome as fair when their own outcome-to-input ratio equals that of a comparative other which is known as equity theory (Adams, 1965). Other distributive rules besides equity concern equality and need perspectives. Equality refers to the belief that resources should be allocated equally across people whereas the need perspective emphasises that resources be allocated according to people's needs (Deutsch, 1975 in Mannix, Neale, \& Northcraft, 1995).

In contrast to these distributive justice theories, more recent research has revealed that people are primarily concerned with the procedure by which the outcome was derived and not with the outcome itself (Lind \& Tyler, 1988; Thibaut \& Walker, 1975; Tyler, 1984). This theory has been referred to as procedural justice. While in the first line of reasoning from that perspective it was argued that people care about procedures because it allows them to have some control over the outcome, later research demonstrated that people care about procedures regardless of the outcome they receive. More specifically, procedural justice was found to have a greater influence on justice perceptions than distributive justice (Lind \& Tyler, 1988; Van den Bos, Wilke, Lind, \& Vermunt, 1998; Thibaut \& Walker, 1975; Tyler, 1984; Tyler, 2006; Wemmers, van der Leeden, \& Steensma, 1995). People were found to regard an outcome as fairer if the procedure used to obtain the outcome was fair regardless of the favourability of the outcome. Moreover, people were more satisfied with an unfavourable outcome if the procedure used to obtain it was perceived as fair (Tyler, 1984; Tyler, 1996).

The dichotomy between distributive and procedural justice was first introduced by Thibaut and Walker (1975). They established two criteria of justice: decision control and process control. Decision control refers to the amount of influence an individual has on the outcome whereas process control refers to the amount of influence an individual has on the process by which the outcome is derived. Research demonstrated that people were 
willing to dismiss decision control as long as they retained process control (Thibaut \& Walker, 1975). Thibaut and Walker (1975) believed that in situations of third-person interventions, in which people have to abdicate some of their control, people try to exert control indirectly over the decision by process control. They concluded that people are primarily interested in receiving a favourable outcome and that they use process control as an indirect means to control decisions. This conclusion was interpreted as a fundamental weakness of their theory as later research demonstrated that people valued process control irrespective of the outcome they received (Lind \& Tyler, 1988; Tyler, 1984). Nevertheless, the findings of Thibaut and Walker (1975) regarding the dichotomy between distributive and procedural justice was rather unexpected and led other researchers to further analyse this effect and potential antecedents of procedural justice in order to be able to identify and determine specific aspects that make a procedure be viewed as fair (Bies \& Moag, 1986; Bies \& Shapiro, 1988; Leventhal, 1980; Lind \& Tyler, 1988; Tyler, 1984; Tyler, 1988, Tyler, 1994; Van den Bos, Vermunt, \& Wilke, 1997; Van den Bos, Wilke, Lind, \& Vermunt, 1998). The various indicators of procedural justice are discussed in more detail in the following section.

\subsection{Indicators of procedural justice}

Several researchers were interested in knowing when people view a procedure as fair and have therefore investigated which aspects of procedures indicate procedural justice (e.g. Bies \& Shapiro, 1988; Lind \& Tyler, 1988; Tyler, 1984; Tyler, 1988; Tyler \& Folger, 1980). One of the most replicated findings is that people perceive procedures as fair if these procedures allow them voice. Voice procedures give people the opportunity to present their case. In addition to voice, other antecedents of procedural justice have been identified. These antecedents have been studied in various contexts. While Thibaut and Walker (1975) examined justice perceptions in the legal context, other researchers analysed justice perceptions in organisational settings (Colquitt, 2001; Folger, 1987; Leventhal, 1980). These two settings are similar in that an authority person, who in the legal context is the neutral person deciding the outcome and in the organisational context the 
employer, has control over the process and its outcome and determines the degree of control he allows the litigant and defendant or his employee respectively. In both settings the participants have concerns about fairness. Before a court, the litigant wants to be able to present his case and receive an outcome that is based on accurate information. In addition, the neutral person wants the two parties to accept and comply with his decision. In an organisation, the employee wants his performance to be appraised on the basis of correct information, being able to submit information and adjust misimpressions. Additionally, the employer wants his employees to be satisfied with outcomes and to be dedicated and loyal. It might be argued that contextual characteristics influence the relative importance of indicators of procedural justice in distinct settings. Nevertheless, as fairness plays a role in all social situations and it has been stated that the same basic psychological processes occur in different procedural contexts it may be inferred that basically the same indicators are important in different procedural contexts (Lind \& Tyler, 1988). In fact, the same indicators of procedural justice that were found to be relevant in the legal context were also found to be relevant in the organisational context and vice versa (e.g. Colquitt, 2001; Folger, 1987; Lind \& Tyler, 1988; Lind et al., 1990; Thibaut \& Walker, 1975). Many of the recent studies have focused on procedural justice in the organisational context. Because of the aforementioned similarities between the two settings and the fact that indicators of procedural justice seem to be contextindependent, the findings of research on organisational justice are also relied on for the purpose of this research project and are therefore included in the discussion on important research findings of procedural justice research.

As previously mentioned, Thibaut and Walker (1975) made a distinction between decision and process control. While they examined justice perceptions in the legal context, Leventhal (1980 in Tyler, 2006) focused on justice perceptions in organisational settings. He was among the first to propose six specific criteria that a procedure should meet if it is to be perceived as fair. These criteria include consistency (i.e. procedures are applied consistently across people and across time), bias suppression (i.e. the decision-maker should be neutral), accuracy (i.e. procedures are based on accurate information), correctability (i.e. appeal procedures for correcting 
inaccurate outcomes), representation (i.e. procedures allow control at every stage of the process), and ethicality (i.e. the procedure implements general ethical and moral standards). By proposing these criteria, Leventhal (1980) moved beyond issues of control as a basis for evaluations of justice as had been emphasised by Thibaut and Walker (1975). It has been argued that there is some overlap between the concept of representation and the control items defined by Thibaut and Walker (1975). Although the concept of representation has never been clearly defined by Leventhal (1980) it is believed to be equal to decision and process control (Colquitt, 2001; Lind \& Tyler, 1988). Accordingly, the two concepts have been used interchangeably.

The criteria proposed by Leventhal (1980) have been evaluated in several studies resulting in the finding that they are fairly accurate (Lind \& Tyler, 1988; Tyler, 1988). Apparently, people view procedures as fair if these procedures allow them to present their case, in which the decision-maker is unbiased and neutral and relies on accurate information when deriving an outcome. Furthermore, the decision-maker needs to be consistent and to consider ethical and moral standards. The individual using a procedure should have the right to correct statements and to appeal against an unfavourable decision.

The importance of procedural justice has not only been recognised by social psychologist but also by theorists and practitioners with a legal background as various indicators of procedural justice are incorporated into the law. Some of the indicators of procedural and interactional justice that have been identified within the scope of social psychology, correspond to rules of procedure such as accuracy, neutrality, and participation (Crombag \& Van Koppen, 2002; Solum, 2004), as well as to the postulates included in Article 6 of the European Convention for the Protection of Human Rights (ECHR) which declares that "everyone is entitled to a fair and public hearing within a reasonable time by an independent and impartial tribunal". Furthermore, judgment should take into account interests of morals and public order. These postulates correspond to the aforementioned indicators of procedural justice. Voice, decision and process control, bias suppression and ethicality are incorporated in Article 6 of the ECHR. The fact that several antecedents which social psychology research has found to indicate 
procedural justice are identified as significant postulates by professionals with a legal background demonstrates the importance and apparent universality of these antecedents. Since the focus here is on insights from social psychology research legal perspectives are not discussed in more detail.

In addition to indicators of procedural justice, aspects pertaining to the interactional treatment people receive when procedures are enacted were found to influence justice judgments. Specifically, Bies and Moag (1986 in Colquitt, 2001) introduced the concept of interactional justice. People want to be treated with respect and dignity and view procedures as unfair if they do not recognise their status as a valuable member of society. Additionally, justice perceptions were found to be influenced by the amount and content of information people receive regarding the procedure and the allocation of outcomes. Apparently, providing explanations about the procedure and outcome increases people's perceptions of fairness and is hence likely to result in higher levels of cooperation with the authority. Providing information may also play a role at earlier stages in a process since some procedures may be cognitively demanding especially for laypersons and hence providing people with information about several aspects of the process seems beneficial with regard to people's justice perceptions (Bies \& Shapiro, 1987; Colquitt, 2001; Lane, 1988; Shapiro, Buttner, \& Barry, 1994). The two types of interactional justice have been referred to as interpersonal and informational justice respectively. Empirical studies have demonstrated that procedures in which people are treated with respect and politeness and in which the rationales of procedures and decisions are explained result in more favourable justice perceptions (Colquitt, 2001; Bies \& Shapiro, 1987; Bies \& Shapiro, 1988). In contrast, any perceived violation of these aspects of interactional justice or the aforementioned aspects of procedural justice has a negative impact on justice evaluations regardless of the favourability of outcome.

While it is generally agreed that interpersonal aspects such as honesty and respect and informational aspects such as truthfulness and justification influence people's justice judgments, there is an ongoing debate about the conceptualisation of justice. More specifically, it is unclear whether interactional justice, including interpersonal and informational justice, is a third 
type of justice in addition to distributive and procedural justice or whether it is a form of procedural justice. The distinct dimensions of justice are discussed in more detail in section 2.3. The following section deals with the question why people find procedural justice important.

\subsection{Why people value procedural justice}

The finding that people's justice perceptions are primarily influenced by aspects of procedural justice is remarkable. Intuitively, one may be inclined to think that somebody who takes legal action in order to obtain a certain outcome does not care much about the procedure as long as he receives a favourable decision. However, people care about the procedure that is used to derive an outcome and regard an outcome as fairer if the procedure used to obtain it is perceived as fair (Tyler, 1984). Moreover, if people received an unfavourable outcome they were found to be more satisfied if the procedure used to obtain the outcome was fair (Lind \& Tyler, 1988). Research has demonstrated that variables related to procedural justice explain more variance in justice judgments than variables related to distributive justice (Lind, Kulik, Ambrose, \& De Vera Park, 1993; Tyler, 1984, 1988). Several theoretical frameworks for the fair process effect have been proposed. Some of these are addressed in the following sections.

\subsubsection{Referent cognition theory}

Referent cognition theory is based on what is known from research on reasoning and decision-making. According to referent cognition theory, people's evaluations of justice depend largely on counterfactual thinking. This means that people have a framework of reference which they use in order to evaluate whether the procedure and outcome they obtained was fair and whether they could have obtained a fairer procedure or outcome instead (Folger, 1987; Van den Bos \& Van Prooijen, 2001). People are unsatisfied with their outcome if they feel that they could have obtained another outcome and if they feel that aspects of the procedure prevented this better outcome from being obtained (Folger, 1987). People were found to show the highest levels of discontent when they experienced an unfair procedure and had a 
high referent outcome (Folger, 1987; Van den Bos \& Van Prooijen, 2001). Hence, when people view a procedure as unfair and know that they could have obtained a fairer procedure if certain aspects of procedural justice were followed they are very likely to be unsatisfied with the procedure and the outcome.

The assumptions of referent cognition theory can be applied to the legal context. If a person receives an unfavourable outcome and feels that a procedural rule has been broken he will be more dissatisfied if he believes that there was an alternative situation in which he would have received a more favourable outcome. Within the legal context, an alternative situation might simply be another authority which in the belief of the person receiving the unfavourable outcome, might have applied a fairer procedure.

\subsubsection{Relational models}

Another explanation for the finding that people value procedural justice highly has been provided by the group-value model and the relational model (Lind \& Tyler, 1988; Tyler, 1994). More recently, these two models have been integrated in the group-engagement model (Blader \& Tyler, 2003). According to these models, procedural justice is important to people because it indicates the degree to which people are valued by authorities and the group to which they belong. According to these models, people are primarily concerned with their long-term social relationship with authorities and institutions that employ procedures. Procedures implicitly convey information about a person's status in the group, and hence serve as an important determinant of feelings of value to the group. The two models emphasise the importance of interpersonal treatment by focusing on an individual's experience of enhanced social standing in a group as determined by the perceived treatment of the authorities as a basis for procedural justice judgments. Procedural justice judgments are hence for a large part based on how one is treated by the authorities and how this treatment is evaluated in the light of one's social standing in and belongingness to a group. Several studies have demonstrated that relational aspects of fair procedures, including neutrality and trustworthiness, communicate group-relevant information to individuals (Tyler, 
1989; Tyler, Degoey, \& Smith, 1996). In a recent study, it was demonstrated that manipulating people's status salience had an effect on people's fairness evaluations (Van Prooijen, Van den Bos, \& Wilke, 2002). More specifically, if people were aware of their status in a group this resulted in more attention being paid to fairness issues. Apparently, when people are aware of their status in a group they react more strongly to perceived procedural justice and injustice.

Within the legal context both feelings of exclusion and exploitation play an important role. Feelings of social standing refer to how one is treated by the authority with regard to general rules and standards. Feelings of exploitation play a role in the sense that the authority could behave or decide in an unfair and unfavourable way. If a legal system warrants that people who enter into a legal procedure are treated honestly and respectfully and that procedures are impartial, accurate and consistent, any perceived violation of these standards will result in the feeling that the authority does not accord them the appropriate standing as a member of society. This, in turn, may lead to more negative feelings and self-evaluations and may consequently result in less cooperation with the authority. Interestingly, recent research has revealed that procedural fairness is negatively correlated with self-evaluations when outcomes are unfavourable if people consider unfavourable outcomes as psychologically significant, i.e. if they focus on perceived losses rather than perceived gains (Brockner, De Cremer, Fishman, \& Spiegel, in press). This finding suggests that people's self-evaluations are influenced by more than just procedural justice information. It is nevertheless important to notice thatone of the main reasons why people's fairness evaluations are strongly influenced by procedural justice perceptions concerns the fact that the authority's behaviour conveys information about the value and social standing of an individual.

Procedural justice evaluations convey information about an individual's social status and membership to a group. If procedures are fair people feel themselves to be valued members of a group and are likely to commit to the group. Within the legal context this means that an individual who feels that he is being treated as a valued member of a group is likely to accept and comply with a decision that is reached by a fair procedure. 


\subsubsection{Fairness heuristic theory}

In an attempt to integrate the distributive and procedural justice domains and explain the finding of numerous studies demonstrating that procedural justice has a greater influence on overall justice judgments than distributive justice, the fairness heuristic theory (Van den Bos, Vermunt, \& Wilke, 1996, 1997; Van den Bos, Lind, Vermunt, \& Wilke, 1997; Van den Bos, Wilke, \& Lind, 1998; Van den Bos, Wilke, Lind, \& Vermunt, 1998) has been developed. This perspective is based on the aforementioned group-value and relational model and states that people use their judgments of fairness as a heuristic to evaluate the fairness of outcomes and procedures and depend on that to make decisions about their subsequent reactions. Hence, within the legal context people may use perceptions of fairness as a heuristic to decide whether to accept and comply with the authority's decision. People are believed to use fairness heuristics in situations in which they run the risk of exclusion or exploitation.

It has been stressed that one of the major contributions of the fairness heuristic theory pertains to its emphasis on the relevance of the kind and amount of information available to people when they make a justice judgment. When evaluating justice people search for the most relevant information for their current situation. If the most relevant information is not available people use other information to evaluate fairness and determine their reaction to the situation. Hence, the less relevant but available information serves as the fairness heuristic people use to judge justice. An important situation that highlights this effect is closely linked to distributive justice. As previously discussed, equity theory states that people judge an outcome as fair if their outcome-to-input ratio equals that of a comparative other. However, it has been argued that people often do not know the outcome of comparative others (Van den Bos, Lind, Vermunt, \& Wilke, 1997). In these uncertain situations, the most relevant information to judge fairness is lacking and people have to rely on other information, i.e. information about procedural justice, in order to assess fairness. Research supports this prediction of the fairness heuristic theory (Van den Bos, Vermunt, \& Wilke, 1997; Van den Bos, Wilke, \& Lind, 1998; Van den Bos, Wilke, Lind, \& Vermunt, 1998). When people know the outcome of a comparative other, their fairness judgments do 
not differ as a function of procedural justice (Van den Bos, Lind, Vermunt, \& Wilke, 1997). Similar effects have been found for people's outcome expectations, their knowledge of the authority's trustworthiness and whether or not people receive voice (Van den Bos, Wilke, Lind, \& Vermunt, 1998; Van den Bos, Wilke, \& Lind, 1998; Van den Bos, Wilke, Lind, \& Vermunt, 1999). Moreover, within the legal context information about the procedure is available to a person prior to information about the outcome which further supports the notion that people rely on procedural justice when they make overall justice judgments. The fact that people generally tend to hold on to their early impressions is likely to at least partly account for the finding that procedural justice has a greater effect on justice perceptions than distributive justice. In addition, it has been argued that in contrast to outcome information, procedural information is less ambiguous and easier to interpret by the individual (Lind et al., 1993).

According to fairness heuristic theory, authority processes such as legal procedures are important conditions in which fairness becomes crucial to an individual because in these situations people have to rely on the authority which entails the possibility of exclusion and exploitation. People typically do not know whether the authority is trustworthy and whether they will receive a procedure characterised by voice, consistency, bias suppression, accuracy, correctability and ethicality. In addition, they often do not know the outcome of comparison others who may have had a similar conflict and they may even find it difficult to have a clear outcome expectation. Moreover, in a legal procedure people do not know the outcome before they know the procedure. Hence, as predicted by fairness heuristic theory, people need a heuristic substitute in order to judge fairness. Procedural justice seems to be the most important indicator for overall fairness perceptions in situations of uncertainty which legal procedures most probably are for most people.

The three theoretical frameworks described above - referent cognition theory, the relational models and fairness heuristic theory - highlight different functions of procedural justice. All three theories have received empirical support. It therefore seems beneficial to view them as complementary rather than exclusionary. 


\section{Conceptualisations of justice}

As previously mentioned, there is an ongoing debate about the conceptualisation of justice. More specifically, it is still unclear whether justice is best depicted by two (distributive and procedural justice), three (distributive, procedural and interactional justice) or by four factors (distributive, procedural, interpersonal and informational justice).

It is generally agreed that distributive and procedural justice are two distinct dimensions of justice. Research suggests that different types of justice have different effects on attitudes (Colquitt, 2001; Colquitt et al., 2001; Sweeney \& McFarlin, 1997). Distributive justice was found to be more related to specific events such as outcome satisfaction whereas procedural justice was found to be related to evaluations of a system or organisation. Hence, procedural justice provides information about the rules of relationships and is therefore an important determinant of people's attitudes towards a system or organisation. Within the legal context, distributive justice is hence related to a person's satisfaction with the outcome of the procedure, e.g. the judge's decision, whereas procedural justice is related to a person's satisfaction with the judicial system in general. Despite these findings, several studies have found high correlations between the two concepts (Folger, 1987; Sweeney \& McFarlin, 1997). It has therefore been argued by some researchers that attitudes that are affected by distributive justice mediate the relationship between all types of justice and system-related attitudes (Ambrose, Hess, \& Ganesan, 2007). The implications of this research suggest that distributive justice effects are more important than previously assumed.

The discussion about the conceptualisation of justice has become even more complicated after the introduction of interactional justice (Bies \& Moag, 1986 in Colquitt, 2001; Bies \& Shapiro, 1986). Interactional justice has been defined as the quality of the interpersonal treatment that people receive when procedures are implemented. More recently, it has been argued that the interactional dimension of justice actually consists of two distinct facets: interpersonal and informational justice (Greenberg, 1993 in Colquitt, 2001).

Interpersonal justice is defined as the degree to which people are treated with politeness, dignity and respect by the authority in question. Several studies have analysed interpersonal aspects of justice resulting in the finding 
that the way people are treated by the authority influences their justice evaluations (Colquitt, 2001; Lind et al., 1990; Wenzel, 2006). Moreover, research suggests that dignity, being an aspect of interpersonal justice, is the crucial variable influencing people's fairness perceptions (Lind et al., 1990). Additional support for the notion of interpersonal justice comes from the theoretical and empirical work on the relational models of justice as described in the previous paragraph. According to these models, the treatment people receive is central to their evaluations of justice.

Informational justice refers to the explanations and justifications provided to people. These should convey information about both procedures and outcomes. Research findings indicate that informational justice is an important factor in the psychological process of forming justice judgments (Bies \& Shapiro, 1987; Wenzel, 2006). Informing people about general rules and their rights in a procedure before applying these was found to have a positive effect on justice perceptions (Van den Bos, Vermunt, \& Wilke, 1996). In addition, explanations influence fairness perceptions because they may convey the impression that a decision has been based on accurate information and that the authority has acted in an unbiased, consistent and reasonable way. Providing an explanation generally contributes to the impression that one is being treated with respect and with regard to ethical values (Bies \& Shapiro, 1987; Wenzel, 2006). Moreover, in certain situations a causal account may suggest that an outcome was derived because of the circumstances and not because of the individual's responsibility. In contrast, if no explanations are provided a person may infer that a certain decision was obtained or certain procedures were applied in order to intentionally disadvantage him or her (Bies \& Shapiro, 1987).

This is in line with what has previously been proposed by the relational models of justice. If a person feels that he is being unfairly treated, not receiving an adequate explanation for certain actions, he will feel that he is resented by the person implementing the procedure which is likely to result in a refusal to cooperate with the authority's decision. Research has furthermore demonstrated that the mere providing of a causal account is not sufficient. In evaluating informational fairness, people were found to rely more on the 
adequacy of the explanation than the mere fact that they received an explanation (Bies \& Shapiro, 1987; Shapiro, Buttner, \& Barry, 1994).

So far, most researchers have operationalised justice by measuring distributive justice, on the one hand, and procedural justice in combination with interactional justice on the other. In this view, interactional justice is not a separate dimension of justice but a social form of procedural justice along with the structural aspects of procedural justice such as the Leventhal criteria. While it is generally agreed that various indicators of justice, including aspects of procedural justice such as voice, consistency and accuracy as well as aspects of interactional justice including honesty, respect and truthfulness influence people's justice perceptions, there is no consensus about whether interactional justice is a separate justice dimension. A review of the literature on the distinct criteria people use to evaluate the fairness of procedures revealed that there is considerable variation across studies in how justice is operationalised and measured. Different conceptualisations of justice have been applied by distinct researchers. As a result it is sometimes unclear whether the factors measured by different researchers are actually distinct or whether they combine the same factor. Moreover, the same indicators have been used by different researchers to measure distinct justice dimensions. As previously described this not only complicates valid comparisons of research findings, but it also leads to the inference that it is unclear whether justice is best depicted by two, three or even four distinct factors.

Some more recent research has however suggested that distributive procedural, interpersonal and informational justice are distinct forms of justice (Cohen-Charash \& Spector, 2001; Colquitt, 2001; Colquitt et al., 2001). These four dimensions of justice are believed to have differential effects on people's attitudes. As previously mentioned, distributive justice affects attitudes about specific events and procedural justice affects system-related attitudes. Interpersonal justice was found to affect person-related attitudes, such as trust in and satisfaction with the authority. Informational justice is related to attitudes about the system or organisation especially with a person's commitment to that system or organisation (Cohen-Charash \& Spector, 2001; Colquitt, 2001). 
Colquitt (2001) explored the dimensionality of organisational justice. He tested whether a four-factor solution that takes distributive, procedural as well as interpersonal and informational justice into account is a more complete conceptualisation of justice than one that combines distinct types of justice into one, two or three factors. The distinct factor structures were compared in order to test the theoretical dimensionality of justice resulting in the finding that the four-factor solution best fits the data. The construct and predictive validity of the measure were analysed yielding satisfactory results. Furthermore, the research findings supported previous suggestions by demonstrating that the four distinct justice dimensions had differing effects on people's attitudes. The results indicated that distributive justice was related to outcome satisfaction. In contrast, procedural justice was found to affect rule compliance, which is defined as the degree of adherence to guidelines that govern a system or organisation. Interpersonal justice was found to be related to evaluations of authority figures and informational justice was found to be related to collective esteem, i.e. the extent to which a person feels he is valued by a group. The finding that the four distinct facets of justice have independent effects on justice perceptions suggests that they need to be differentiated from one another. Disregarding either of the four dimensions results in an incomplete conceptualisation of justice. In addition, important differences between the distinct concepts would remain unnoticed when joining procedural and interactional justice together.
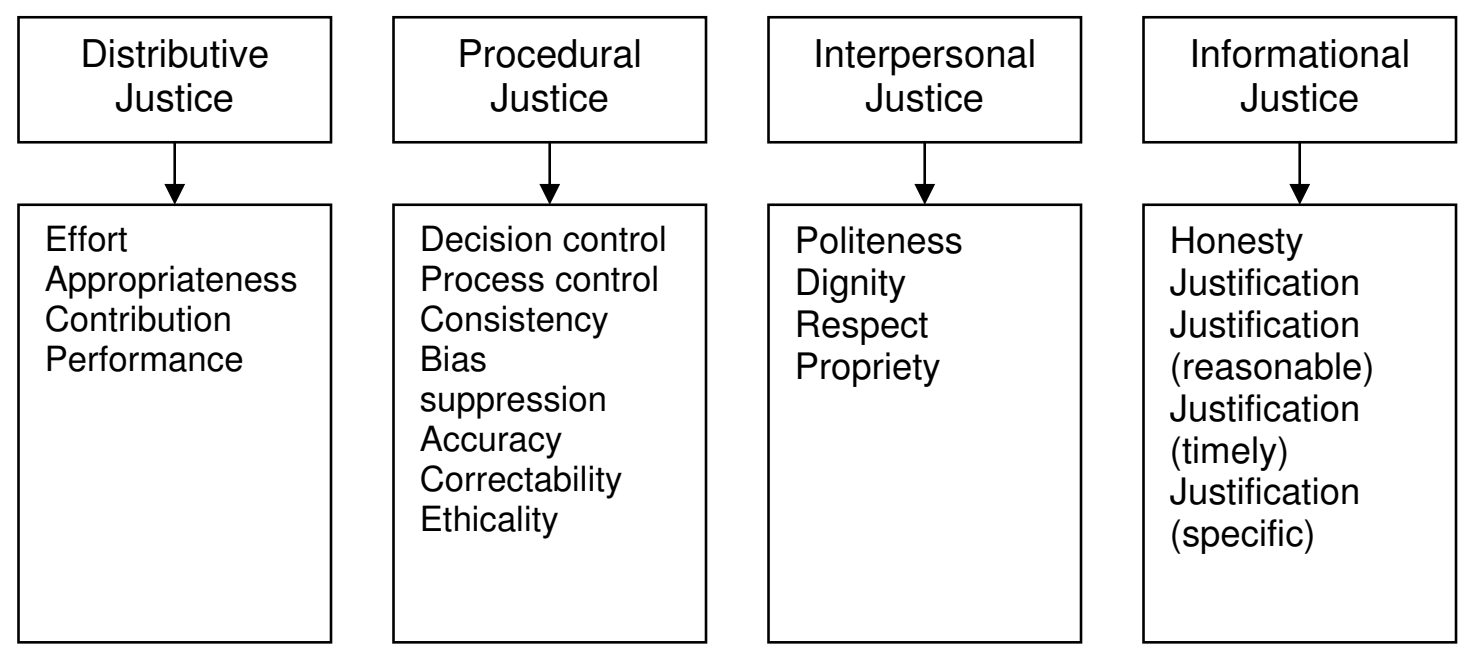

Figure 1. Four-factor model of justice (Colquitt, 2001). 
The distinct indicators used in the measure developed by Colquitt (2001) where derived from previous research. Figure 1 shows which indicators were used to measure the four justice factors. Distributive justice is operationalised by measuring effort, appropriateness, contribution and performance. Decision control, process control, consistency, bias suppression, accuracy, correctability and ethicality were combined to measure procedural justice. The measure includes decision and process control as proposed by Thibaut and Walker (1975), and the Leventhal criteria with the exception of representation as this was argued to be covered by the control items of Thibaut and Walker (1975). Interpersonal justice was operationalised by measuring politeness, dignity, respect and propriety. These items had been identified in previous research by Bies and Moag (1986 in Colquitt, 2001). Informational justice was operationalised by measuring honesty and three distinct aspects of justification. These criteria were derived from research by Bies and Moag (1986 in Colquitt, 2001) and Shapiro and her colleagues (1994).

The measure developed and validated by Colquitt (2001) applies to justice perceptions in the organisational context. As previously mentioned, similar results pertaining to the influence of the various antecedents of procedural, interpersonal and informational justice factors have been obtained by research across various settings. Since there are several analogies between justice perceptions in the legal and the organisational context, it is assumed that a conceptualisation of justice that has been validated within the organisational setting can be applied to the legal context. However, it needs to be mentioned that research demonstrating that the four-factor solution also best applies to data from the legal context is still lacking. Nevertheless, the Colquitt model is believed to be the most complete and representative conceptualisation of justice and is consequentially adopted for the purpose of the present project. 


\begin{tabular}{|c|c|c|}
\hline Indicator & Justice type & Description \\
\hline Process control & Procedural justice & $\begin{array}{l}\text { Amount of influence an individual has on } \\
\text { the process }\end{array}$ \\
\hline Decision control & Procedural justice & $\begin{array}{l}\text { Amount of influence an individual has on } \\
\text { the outcome }\end{array}$ \\
\hline Consistency & Procedural justice & $\begin{array}{l}\text { Procedures are applied consistently } \\
\text { across people and across time }\end{array}$ \\
\hline Bias suppression & Procedural justice & $\begin{array}{l}\text { Decision-maker should be neutral in order } \\
\text { to ensure that procedures are free from } \\
\text { bias }\end{array}$ \\
\hline Accuracy & Procedural justice & $\begin{array}{l}\text { Procedures should be based on accurate } \\
\text { information }\end{array}$ \\
\hline Correctability & Procedural justice & $\begin{array}{l}\text { There should be a possibility to correct or } \\
\text { contradict statements and there should be } \\
\text { appeal procedures for correcting } \\
\text { inaccurate outcomes }\end{array}$ \\
\hline Ethicality & Procedural justice & $\begin{array}{l}\text { Procedure should implement general } \\
\text { ethical and moral standards }\end{array}$ \\
\hline Politeness & Interpersonal justice & Authority should treat people politely \\
\hline Dignity & Interpersonal justice & Authority should treat people with dignity \\
\hline Respect & Interpersonal justice & Authority should treat people with respect \\
\hline Propriety & Interpersonal justice & $\begin{array}{l}\text { Authority should refrain from improper } \\
\text { remarks or comments }\end{array}$ \\
\hline Honesty & Informational justice & $\begin{array}{l}\text { Authority should be truthful in its } \\
\text { communications }\end{array}$ \\
\hline Justification & Informational justice & $\begin{array}{l}\text { Authority should provide explanations } \\
\text { about procedure }\end{array}$ \\
\hline $\begin{array}{l}\text { Reasonable } \\
\text { justification }\end{array}$ & Informational justice & $\begin{array}{l}\text { Explanations regarding procedure should } \\
\text { be reasonable }\end{array}$ \\
\hline Timely justification & Informational justice & $\begin{array}{l}\text { Details about procedure should be } \\
\text { communicated in a timely manner }\end{array}$ \\
\hline Specific justification & Informational justice & $\begin{array}{l}\text { Explanations should be tailored according } \\
\text { to the individual's specific needs }\end{array}$ \\
\hline Effort & Distributive justice & $\begin{array}{l}\text { The outcome should reflect the effort put } \\
\text { into the case }\end{array}$ \\
\hline Appropriateness & Distributive justice & $\begin{array}{l}\text { The outcome should be appropriate for the } \\
\text { input }\end{array}$ \\
\hline Contribution & Distributive justice & $\begin{array}{l}\text { The outcome should reflect what the } \\
\text { individual contributed }\end{array}$ \\
\hline Performance & Distributive justice & $\begin{array}{l}\text { The outcome should be justified given the } \\
\text { input }\end{array}$ \\
\hline
\end{tabular}

Table 1. List of justice indicators (Colquitt, 2001).

As described in the introduction, the purpose of the present project is to develop a method that can be used in order to evaluate the quality of procedures. It has been proposed that the quality of a procedure can be assessed by measuring people's perceptions of procedural justice. From the research findings outlined in this section it follows however, that the quality of a procedure is not only influenced by procedural justice but also by aspects of 
interpersonal and informational justice. Therefore, the quality of a procedure is measured by taking these three distinct dimensions of justice into account. Distributive justice is not included as an aspect of the quality of a procedure. This is not to say that distributive justice is not important, especially with regard to the research findings suggesting that it may be a mediating factor on all types of justice, but rather that it is a separate constituent of a legal process ${ }^{4} 5$. Despite the fact that procedural, interpersonal and informational justice are independent types of justice they all refer to the procedure. Procedural justice applies to the structural aspects of a procedure, interpersonal justice to the treatment which a person receives when procedures are implemented and informational justice to the explanations provided to a person while he is partaking in a procedure.

\section{Factors influencing justice judgments}

Several factors were found to influence procedural justice judgments. Research on interactional justice as an independent type of justice has only recently received empirical attention and therefore little is known about factors influencing evaluations of interactional justice. Consequently, the discussion about factors influencing justice judgments is here limited to procedural justice. It may however be assumed that factors that influence procedural justice evaluations also have an effect on interpersonal and informational justice evaluations.

\subsection{Characteristics of the person}

Generally, research investigating the influence of individual factors on procedural justice judgments has found no main effects of gender, age and ethnicity (Kulik, Lind, Ambrose, \& MacCoun, 1996; Lind et al., 1990; Tyler, 1988; Tyler, 2006). This means that the fairness of a procedure is judged by the same criteria regardless of these individual characteristics. However,

\footnotetext{
${ }^{4}$ Distributive justice is included in another project measuring the quality of the outcome and it is therefore not included as a factor of the quality of procedures. It is acknowledged that not considering distributive justice is likely to lead to a misinterpretation of research findings. Therefore, distributive justice as included in the measure developed by Colquitt (2001) has been incorporated in the measure that is developed for the purpose of evaluating the quality of outcomes (see project c "The quality of the outcome").
} 
when analysing the importance that women and men place on distributive and procedural justice gender differences were found (Sweeney \& McFarlin, 1997). Apparently, women pay more attention to process-oriented issues whereas men pay more attention to outcome-oriented issues.

Research has found no link between income and procedural justice perceptions (Lind et al., 1990). Education and political views were found to influence justice perceptions with people who are more highly educated and liberal paying more attention to issues of ethicality (Tyler, 1988).

\subsection{Culture}

Several studies have explored the universality of procedural justice across cultures. Procedural justice seems to be equally important to people from different cultures and procedural justice seems to be defined largely in terms of the same variables across cultures (Brockner et al., 2001; Lind, Tyler, \& Huo, 1997; Price et al., 2001; Sugawara \& Huo, 1994). Some evidence was found for the assumption that people in egalitarian cultures, including Germany and the United States, are more concerned about status recognition while people in hierarchical societies, such as China, care more about trust and neutrality. This can probably be ascribed to inclusion being more important in egalitarian cultures. People in hierarchical societies are believed to worry more about the abuse of power (Lind, Tyler, \& Huo, 1997). Similar results were found for voice. In cultures in which power distance, defined as the extent to which less powerful members of society or an organisation accept and expect that power is distributed unequally, is high, people are more willing to accept lower levels of participation. Hence, in these cultures voice is expected to be less important. Research findings analysing the importance of voice for people from different cultures found that in Mexico, which is a culture that rates higher on power distance than Great Britain and The Netherlands, voice was less important (Price et al., 2001). Moreover, research found that in low power distance cultures, such as the United States and Germany, people respond less favourably to lower levels of voice than in high power distance cultures, such as China, Mexico and Hong Kong (Brockner et al., 2001). 
Taken together, these results suggest that procedural justice is equally important across cultures. However, while the same basic processes involved in evaluations of procedural justice were found in different cultures, the weight attached to certain indicators can vary across cultures. A crucial determinant of this effect seems to be the degree of power distance.

\subsection{Outcome information and outcome favourability}

An important factor influencing procedural justice judgments seems to be the sequence in which people receive information (Tyler, 1996; Van den Bos, Vermunt, \& Wilke, 1997). As already described, people tend to hold on to their earlier impressions which is one explanation for the fact that procedural justice has a greater impact on justice evaluations than distributive justice. However, if people know the outcome when evaluating procedural justice this knowledge is likely to influence the weight people attach to procedural justice. Apparently, people pay less attention to procedural justice if they know the outcome prior to evaluating the fairness of the procedure. Furthermore, this effect was influenced by the favourability of the outcome with more favourable outcomes resulting in less weight being attached to procedural justice. The meaning of the distinct indicators of procedural justice is not influenced by prior knowledge of the outcome (Lind, Kulik, Ambrose, \& De Vera Park, 1993; Tyler, 1996).

Outcome favourability depends on both expectations and the outcomes that comparison others receive. If the outcome one receives falls bellow one's expectations or below the outcome that comparison others receive it is evaluated as unfavourable and people rely on procedural information when making justice judgments. Research indicated that interpersonal comparisons provide a stronger basis for justice evaluations than intrapersonal comparisons (Van den Bos, Wilke, Lind, \& Vermunt, 1998). Hence, people who receive an outcome that is better or worse than expected rely on procedural information more than people who receive an outcome that is worse or better than that of a comparison other.

In legal procedures people typically do not know the outcome before they know the procedure. However, the effect of outcome information needs to be 
taken into consideration with regard to the measuring times. Procedural justice perceptions differ according to whether or not people know their outcome at the time they evaluate fairness. Taking people's expectations and their knowledge of the outcomes of comparison others into account is likely to contribute to a better understanding of people's justice perceptions.

\subsection{Characteristics of the dispute}

Fairness perceptions are influenced by the nature of the dispute as well as the kind of procedure. However, little research focusing on procedural justice judgments in distinct legal settings is available. It has been suggested that generally procedural justice plays an important role in all types of dispute resolution procedures but that the weight being attached to indicators may differ according to the type of procedure. Trust in benevolence was found to be more important in third-party procedures whereas status recognition and neutrality were found to be of greater concern if a dispute was resolved without third-person intervention (Lind, Tyler, \& Huo, 1997).

Research comparing procedural justice perceptions in the context of citizens' experiences with the police and the court showed that fairness evaluations differ in these two situations. While in both situations procedural justice was found to be strongly linked to satisfaction and fairness perceptions, the meaning of procedural justice varied according to the nature of the situation. Procedures used to resolve disputes between contending factions were found to be more likely to be judged in terms of decision control, bias and correctability as opposed to procedures that do not intend to resolve a dispute such as contacts with the police. In these situations people were found to be more concerned with the efforts made (Tyler, 1988).

Research investigating tort litigants' evaluations of their experiences in the civil justice system indicated that fairness perceptions differed between people who had a court trial and those who had an alternative litigation procedure. Objective measures such as outcome, costs and delay were unrelated to procedural justice judgments whereas subjective measures of these indicators, i.e. people's expectations, were highly related to procedural justice. Perceptions of procedural justice were better among people who 
reported that they had experienced a high level of dignity. Moreover, dignity was found to be the crucial factor differentiating trials and bilateral settlement procedures. Trials were viewed as more dignified than bilateral settlement procedures resulting in higher procedural justice ratings. Overall, whether litigants received respectful treatment was the most important variable in their evaluations of procedural fairness (Lind et al., 1990). Apparently, people view trial and arbitration procedures as more dignified than bilateral settlement procedures which led to more favourable justice judgments. Procedures that provide a quicker and less expensive means to resolve a conflict are not by definition perceived as fairer. It seems that people are more concerned with other aspects of procedures such as interpersonal treatment.

On the basis of the relational model, Wemmers and her colleagues (1995) investigated which criteria crime victims use when judging fairness. The results of their study indicated that within this particular context, interpersonal indicators of justice are of particular importance. Victims were found to be particularly concerned about being treated with dignity and respect and receiving the opportunity to present their case. Outcome information was found to have very little influence on justice perceptions (Wemmers, Van der Leeden, \& Steensma, 1995).

Other contextual factors are also believed to exert an influence on procedural justice judgments. In this regard, it seems likely that a personal stake plays an important role in justice judgments with procedural justice being more important when a personal stake is high and when the relationship with the other party is important such as e.g. in divorce cases. However, research suggests that the link between personal stake and procedural justice perceptions is more complex. The results of a recent study indicated that people who receive an unfavourable outcome in a context in which they feel they are strongly evaluated on the basis of their performance or characteristics are more inclined to search for an explanation for their unfavourable outcome (Van den Bos, Bruins, Wilke, \& Dronkert, 1999). Consequently, when the procedure was unfair people reacted more positively than when the procedure was fair. At first sight this finding is counterintuitive as it contradicts the fair process effect which states that people who receive an unfavourable outcome but experience a fair procedure nevertheless react 
more positively as compared to people who experience an unfair procedure. However, the fact that in some situations people feel that they are strongly evaluated results in a search for an explanation for an unfavourable outcome. In these situations, an unfair procedure provides an opportunity to attribute the unfavourable outcome to an external cause. Conversely, fair procedures cannot serve this purpose. Hence, people were found to react more positively after unfair as opposed to fair procedures if their outcome was unfavourable which led the authors to conclude that 'sometimes unfair procedures have nice aspects' (Van den Bos, Bruins, Wilke, \& Dronkert, 1999. p. 334). This effect is more pronounced in a context in which people feel they are strongly evaluated.

It may therefore be concluded that in legal disputes in which an individual's personal involvement is high in terms of his or her personal contribution, as may be the case for example in an employment or divorce case the effect of procedural fairness may be reversed if the outcome is unfavourable. This means that in certain cases, those in which self-relevant processes are strongly triggered, people are more likely to attribute an unfavourable outcome to an unfair procedure. If the procedure was not unfair this external attribution is not possible resulting in a more negative reaction to the outcome. Within the legal context a negative reaction may translate to less cooperation in terms of the acceptance of and compliance with the outcome. This research finding clearly demonstrates the complexity of the psychological processes underlying justice judgments and emphasises the fact that justice judgments cannot be evaluated irrespective of taking both the outcome of a case and the characteristics of that case itself into account.

\section{Emotional aspects of access to justice}

The vast majority of research analysing the link between justice perceptions and emotions has focused on the positive and negative consequences of experiencing justice and injustice respectively (e.g. Krehbiel \& Cropanzano, 2000; Mikula, Scherer, \& Athenstaedt, 1998; Tepper, 2001, Vermunt \& Steensma, 2003). Perceived procedural justice was found to lead to positive emotions whereas perceived injustice was found to lead to negative emotions 
(Krehbiel \& Cropanzano, 2000; Mikula, Scherer, \& Athenstaedt, 1998; Tepper, 2001; Vermunt \& Steensma, 2003; Van den Bos, 2005). Negative emotions resulting from unfair treatment or unfavourable outcomes may include anger, frustration and more generally distress. In line with previous argumentations, distributive and procedural justice were found to have compensatory effects on the experience of psychological distress with people who receive an unfavourable outcome but are still treated procedurally fair, being unlikely to undergo much psychological distress (Tepper, 2001; Vermunt \& Steensma, 2003).

Recent research has indicated that the link between affect and justice perceptions is mutual. A person's emotional state was found to influence his evaluations of fairness (Van den Bos, 2003). Within the legal context this finding may be of eminent importance for two reasons. First, people who embark on legal action by definition have a conflict that needs resolution by a third person. Conflict is generally likely to be accompanied by negative feelings. In addition, relational costs determined as the potential loss of important relationships following from taking legal action is likely to cause feelings of distress. Second, most people are probably unfamiliar with legal procedures and do not know exactly what to expect from the procedure. In order to have their conflict resolved people have to rely on a neutral person. Although people may expect the authority to be trustworthy and unbiased, to act in a consistent and accurate way, and to treat them with politeness and respect, they do not know this with certainty. In addition, they do not know the outcome prior to embarking on a procedure. This is likely to cause feelings of uncertainty and uncontrollability. People may worry about possible complications and potential risks. Hence, apart from the conflict itself, aspects of the procedure are likely to cause feelings of distress. It may therefore be assumed that people who embark on legal action are in a negative emotional state characterised by feelings of stress which is likely to have an effect on how they perceive and evaluate fairness. In addition, negative emotions may be particularly powerful which implies that within the legal context justice perceptions are likely to be negatively affected. Research on the effect of emotional distress on justice judgments within the legal context and whether this effect can somehow be absorbed is still unavailable. The influence of 
affect on procedural justice evaluations within the legal context are incorporated in the present project and will be evaluated in more detail in a follow-up project. The potential role of affect as an antecedent of procedural justice is of particular importance in order to understand the meaning of justice perceptions and it is therefore surprising that apart from the study of Van den Bos (2003) the role of emotions as an antecedent of procedural justice has received only very limited research attention.

Two lines of reasoning from the procedural justice literature support the idea that emotional distress has an influence on people's justice perceptions. First, according to the relational models (Lind \& Tyler, 1988; Tyler, 1994), procedural justice matters to people because they can use it as a source of information to determine their self-identity and social status in a group. Uncertainty about the treatment people receive from the authorities especially with regard to interpersonal justice is likely to be threatening in terms of potential damage to an individual's self-identity and social status appraisal. This potential threat is likely to result in or be accompanied by feelings of distress. Second, the stress literature implicates a lack of control in the experience of psychological distress (Tepper, 2001). Hence, antecedents of procedural justice that denote control, such as decision and process control as well as voice and correctability, are likely to have a rather strong effect on the experience of psychological distress, with individuals who are denied control perceiving higher levels of threat and are hence more likely to experience symptoms of psychological distress. Moreover, at the beginning of a process people do not know whether they receive control and therefore levels of stress may be particularly high at this stage. Theories of procedural justice predict that people tend to avoid situations of third person intervention because they have to abdicate some of their control. Although these theories do not offer an explanation for this assumption it seems likely that people do not like to leave control to another person because this causes distress. Within the legal context people must relinquish at least some of their control. This may cause feelings of stress prior to entering into a legal procedure. Decreasing emotional costs prior to a legal procedure is likely to have a positive influence on justice perceptions. It seems feasible to reduce distress 
by providing people with more information leading to less uncertainty and an increased perception of control.

Only recently, research has analysed the role of affect as an antecedent of justice judgments (Van den Bos, 2003). The results revealed that people who received an outcome but did not know the outcome of a comparison other relied on their affective state when judging the fairness of the outcome. People who were in a positive mood viewed the outcome as fairer than people who were in a negative affective state. The same effect was found with regard to perceptions of procedural fairness. People who did not receive voice were found to judge the way they had been treated as more just when they were in a positive as opposed to a negative affective state. Apparently, in situations of uncertainty people refer to information about their affective state in order to evaluate fairness. This effect was found for both distributive and procedural justice. In addition, negative affect was found to have a stronger influence on fairness judgments than positive affect (Van den Bos, 2003).

The findings of Van den Bos (2003) strongly suggest that emotional state is an important antecedent of procedural justice. Moreover, although there is still a paucity of research, it may be assumed that affect also influences interpersonal and informational justice. The finding of Van de Bos (2003) that justice judgments are not only the result of rational-cognitive processes, but can also be influenced by emotional information, seems to be of particular importance to research on access to justice. Within the legal context people are believed to be in an affective state that is characterised by emotional arousal and this in turn is likely to influence justice perceptions. Moreover, as has been outlined by Van den Bos (2003), emotional information may play a more prominent role in justice judgments than previously thought. It seems likely that emotional information has a mediating effect on other factors influencing justice judgments and, consequently, disregarding this factor may have resulted in an overestimation of other factors that were found to influence justice perceptions. Including information about an individual's emotional state in assessments of procedural justice perceptions seems to be beneficial. 


\section{Conclusion and outline for future research}

For the purpose of this project, the quality of a procedure is determined in terms of people's justice perceptions when procedures are enacted. If people view a procedure as fair this is believed to be an indication for a high quality of that procedure. Being able to accurately assess the quality of procedures is likely to allow comparisons of distinct procedures and may ultimately lead to indications as to how to advance certain procedures thereby ultimately improving access to justice. From the empirical findings discussed above it seems that procedural fairness plays an important role in all types of dispute resolution procedures but that the weight attached to antecedents of procedural justice may differ according to the procedure. It may be concluded that taking aspects of the distinct facets of justice as well as the potential influence of several factors such as those described above into account will result in a relatively complete understanding of justice perceptions.

Justice is a multifaceted concept including distributive justice, on the one hand and procedural, interpersonal and informational justice on the other. The distinct dimensions of justice were found to have independent effects on justice evaluations and it is therefore believed that justice should be depicted by these four factors. The implementation of indicators associated with these four justice dimensions in legal procedures is likely to lead to perceived fairness. If people feel they are fairly treated this enhances cooperation with the authority in terms of the acceptance of and compliance with their decisions.

On the basis of the literature review and the above outlined considerations, a questionnaire measuring aspects of procedural, interpersonal and informational justice as proposed by Colquitt (2001) was developed. The measure developed by Colquitt (2001) is based on indicators of justice that were retrieved from previous studies. The measure has been validated in the organisational context and is believed to be equally applicable to the legal context. The questionnaire that has been developed in order to assess the quality of distinct procedures within the legal context is included in the Appendix. As described at the beginning of the present paper, measuring the quality of procedures is part of a broader research project that is supposed to measure the costs of procedures, the quality of procedures and 
the quality of outcomes. The potential influence of distress on procedural justice perceptions which has been emphasised in the present paper is included in the part that measures the costs of procedures. While several factors might have an influence on procedural justice perceptions, emotional costs as well as distributive justice perceptions along with other aspects of the outcome are believed to be of particular relevance for a reliable interpretation of the results and hence a complete understanding of procedural justice perceptions. The questionnaire included in the Appendix is part of a larger questionnaire that will be discussed in more detail in a paper presenting the first results of pilot studies that are conducted in order to provide more insight into the utility of the measure. It is believed that the results of the pilot study will give a preliminary indication of whether or not certain questions that are now included in the questionnaire need to be excluded, reformulated or amended. In addition, more will be known about the link between the four dimensions of justice (procedural, interpersonal, informational and distributive justice) as well as the influence of other factors such as the money and time spend on a procedure and potential emotional costs of access to justice. The outcomes of these pilot studies will be used in order to adapt and complete the questionnaire. 


\section{Appendix}

Satisfaction with the legal system and compliance with rules (Adopted from Colquitt, 2001) (5-point scale)

1. Please indicate your overall impression of the fairness of the procedure.

2. Please indicate how satisfied you are with the legal system.

Satisfaction with the authority (Adopted from Colquitt, 2001) (5-point scale)

1. Please indicate how satisfied you are with the authority.

2. Please indicate how fairly you were treated by the authority.

3. Please indicate how trustworthy you find the authority.

4. Please indicate whether you would rely on the authority to resolve a conflict in the future.

Procedural justice (Adopted from Colquitt, 2001). During the procedure, to what extent: (5-point scale)

1. Have you been able to express your views and feelings during the procedure?

2. Have you had influence over the outcome arrived at by the procedure?

3. Have the same rules been applied to you and the other party?

4. Has the procedure been objective and neutral?

5. Has the procedure been based on accurate information?

6. Have you been able to appeal against the outcome arrived at by the procedure? Did you know that if the outcome of the procedure would not fulfill your needs you had the opportunity to appeal to a higher instance?

7. Has the procedure upheld ethical or moral standards of society? 
Interpersonal justice (Adopted from Colquitt, 2001) During the procedure, to what extent: (5-point scale)

1. Has the neutral person treated you in a polite manner?

2. Has the neutral person treated you with dignity?

3. Has the neutral person treated you with respect?

4. Has the neutral person made any improper remarks or comments?

Informational justice (Adopted from Colquitt, 2001) During the procedure, to what extent: (5-point scale)

1. Has the neutral person been honest in his/her communication with you?

2. Has the neutral person explained the procedure thoroughly?

3. Were the neutral person's explanations regarding the procedure reasonable? Did you understand the explanations?

4. Has the neutral person communicated details about the procedure in a timely manner?

5. Has the neutral person seemed to tailor his/her communication to your specific needs? 


\section{References}

Adams, J. S. (1965). Inequity in social exchange. In L. Berkowitz (Ed.), Advances in Experimental Social Psychology (Vol. 2, pp. 267-299). New York: Academic Press.

Ambrose, M., Hess, R. L., \& Ganesan, S. (2007). The relationship between justice and attitudes: an examination of justice effects on event and systemrelated attitudes. Organizational Behavior and Human Decision Processes, 103, 21-36.

Baumeister, R. F., Bratslavsky, E., Finkenauer, C., \& Vohs, K. D. (2001). Bad is stronger than good. Review of General Psychology, 5 (4), 323-370.

Bies, R. J., \& Shapiro, D. L. (1987). Interactional fairness judgments: the influence of causal accounts. Social Justice Research, 1 (2), 199-218.

Bies, R. J., \& Shapiro, D. L. (1988). Voice and justification: their influence on procedural fairness judgments. Academy of Management Journal, 31 (3), 676-685.

Blader, S. L., \& Tyler, T. R. (2003). A four-component model of procedural justice: defining the meaning of a "fair" process. Personality and Social Psychology Bulletin, 29, 747-758.

Brockner, J., Ackerman, G., Greenberg, J., Gelfand, M. J., Francesco, A. M., Chen, Z. Y., Leung, K., Bierbrauer, G., Gomez, C., Kirkman, B. L., \& Shapiro, D. (2001). Culture and procedural justice: the influence of power distance on reactions to voice. Journal of Experimental Social Psychology, 37, 300-315.

Brockner, J., De Cremer, D., Van den Bos, K., \& Chen, Y. (2005). The influence of interdependent self-construal on procedural fairness effects. Organizational Behavior and Human Decision Processes, 96, 155-167. 
Brockner, J., De Cremer, D., Fishman, A. Y., \& Spiegel, S. (in press). When does high procedural fairness reduce self-evaluations following unfavorable outcomes?: The moderating effect of prevention focus. Journal of Experimental Social Psychology.

Chebat, J.-C., \& Slusarczyk, W. (2005). How emotions mediate the effects of perceived justice on loyalty in service recovery situations: an empirical study. Journal of Business Research, 58, 664-673.

Cohen, S., Kamarck, T., \& Mermelstein, R. (1983). A global measure of perceived stress. Journal of Health and Social Behavior, 24, 385-396.

Cohen-Charash, Y., \& Spector, P. E. (2001). The role of justice in organizations: a meta-analysis. Organizational Behavior and Human Decision Processes, 86, 278-321.

Colquitt, J. A. (2001). On the dimensionality of organizational justice: a construct validation of a measure. Journal of Applied Psychology, 86 (3), 386400.

Colquitt, J. A., Canlon, D. E., Wesson, M. J., Porter, C. O. L. H., \& Ng, K. Y. (2001). Justice at the millennium: a meta-analytic review of 25 years of organizational justice research. Journal of Applied Psychology, 86, 425-445.

Crombag, H. F. M., \& Van Koppen, P. J. (2002). Rechtvaardigheid. In P. J. Van Koppen, D. J. Hessing, H. Merckelbach, \& H. F. M. Crombag (Eds.), Het recht van binnen: Psychologie van het recht (pp.775-785). Deventer: Kluwer.

De Cremer, D. (2006). Unfair treatment and revenge taking: the roles of collective identification and feelings of disappointment. Group Dynamics: Theory, Research, and Practice, 10 (3), 220-232.

European Convention for the Protection of Human Rights and Fundamental Freedoms as amended by Protocol No. 11, Council of Europe, 1950. 
Folger, R. (1987). Distributive and procedural justice in the workplace. Social Justice Research, 1 (2), 143-159.

Folger, R., Rosenfield, D., Grove, J., \& Corkran, L. (1979). Effects of "voice" and peer opinions on responses to inequity. Journal of Personality and Social Psychology, 37, 2243-2261.

Fondacaro, M. R., Jackson, S. L., \& Luescher, J. (2002). Toward the assessment of procedural and distributive justice in resolving family disputes. Social Justice Research, 15 (4), 341-371.

Forgas, J. P. (2002). Feeling and doing: affective influences on interpersonal behaviour. Psychological Inquiry, 13, 1-28.

Frey, B. S., Benz, M., \& Stutzer, A. (2004). Introducing procedural utility: not only what, but also how matters. Journal of Institutional and Theoretical Economics, 160, 377-401.

Frey, B. S., \& Stutzer, A. (2005). Beyond outcomes: measuring procedural utility. Oxford Economic Papers, 57, 90-111.

Greenberg, J. (1987). Using diaries to promote procedural justice in performance appraisals. Social Justice Research, 1 (2), 219-234.

Kulik, C. T., Lind, E. A., Ambrose, M. L., \& MacCoun, R. J. (1996). Understanding gender differences in distributive and procedural justice. Social Justice Research, 9 (4), 351-369.

Krehbiel, P. J., \& Cropanzano, R. (2000). Procedural justice, outcome favorability and emotion. Social Justice Research, 13 (4), 339-360.

Lane, R. E. (1988). Procedural goods in a democracy: how one is treated versus what one gets. Social Justice Research, 2 (3), 177-192. 
Lind, E. A., Greenberg, J., Scott, K. S., \& Welchans, T. D. (2000). The winding road from employee to complainant: situational and psychological determinants of wrongful termination claims. Administrative Science Quarterly, 45 (3), 557-590.

Lind, E. A., Kulik, C. T., Ambrose, M., \& De Vera Park, M. V. (1993). Individual and corporate dispute resolution: using procedural fairness as a decision heuristic. Administrative Science Quarterly, 38, 224-251.

Lind, E. A., Maccoun, R. J., Ebener, P. A., Felstiner, W. L. F., Hensler, D. R., Resnik, J., \& Tyler, T, R. (1990). In the eye of the beholder: tort litigants' evaluations of their experiences in the civil justice system. Law and Society Review, 24 (4), 953-996.

Lind, E. A., \& Tyler, T. R. (1988). The Social Psychology of Procedural Justice. New York: Plenum.

Lind, E. A., Tyler, T. R., \& Huo, Y. J. (1997). Procedural context and culture: variation in the antecedents of procedural justice judgments. Journal of Personality and Social Psychology, 73 (4), 767-780.

Mannix, E. A., Neale, M. A., \& Northcraft, G. B. (1995). Equity, equality, or need? The effects of organizational culture on the allocation of benefits and burdens. Organizational Behavior and Human Decision Processes, 63 (3), 276-286.

Mikula, G., Scherer, K. R., \& Athenstaedt, U. (1998). The role of injustice in the elicitation of differential emotional reactions. Personality and Social Psychology Bulletin, 24 (7), 769-783.

O'Hear, M. M. (2007). Plea bargaining and procedural justice. Marquette University Law School Legal Studies Research Paper Series, Research Paper No. 07-02. 
Price, K. H., Hall, T. W., Van den Bos, K., Hunton, J. E., Lovett, S., \& Tippett, M. J. (2001). Features of the value function for voice and their consistency across participants from four countries: Great Britain, Mexico, The Netherlands, and the United States. Organizational Behavior and Human Decision Processes, 84 (1), 95-121.

Shapiro, D. L., Buttner, E. H., \& Barry, B. (1994). Explanations: what factors enhance their perceived adequacy? Organizational Behavior and Human Decision Processes, 58, 346-368.

Solum, L. B. (2004). Procedural justice. University of San Diego School of Law Public Law and Legal Theory Research Paper Series, 2. Retrieved November 2nd, 2007, from http://law.bepress.com/sandiegolwps/pllt/art2/

Sugawara, I., \& Huo, Y. J. (1994). Disputes in Japan: a cross-cultural test of the procedural justice model. Social Justice Research, 7 (2), 129-144.

Sweeney, P. D., \& McFarlin, D. B. (1993). Workers' evaluations of the "ends" and "means": an examination of four models of distributive and procedural justice. Organizational Behavior and Human Decision Processes, 55, 23-40.

Sweeney, P. D., \& McFarlin, D. B. (1997). Process and outcome: gender differences in the assessment of justice. Journal of Organizational Behavior, 18, 83-98.

Tepper, B. J. (2001). Health consequences of organizational injustice: tests of main and interactive effects. Organizational Behavior and Human Decision Processes, 86 (2), 197-215.

Thibaut, J., \& Walker, L. (1975). Procedural Justice: A Psychological Analysis. Hillsdale, NJ: Erlbaum. 
Törnblom, K. Y., \& Vermunt, R. (1999). An integrative perspective on social justice: distributive and procedural fairness evaluations of positive and negative outcome allocations. Social Justice Research, 12 (1), 39-64.

Tyler, T. R. (1984). The role of perceived injustice in defendants' evaluations of their courtroom experience. Law and Society Review, 18 (1), 51-74.

Tyler, T. R. (1988). What is procedural justice?: criteria used by citizens to assess the fairness of legal procedures. Law and Society Review, 22 (1), 103135.

Tyler, T. R. (1989). The psychology of procedural justice: a test of the groupvalue model. Journal of Personality and Social Psychology, 57 (5), 830838.

Tyler, T. R. (1994). Psychological models of the justice motive: antecedents of distributive and procedural justice. Journal of Personality and Social Psychology, 67 (5), 850-863.

Tyler, T. R. (1996). The relationship of the outcome and procedural fairness: how does knowing the outcome influence judgments about the procedure? Social Justice Research, 9 (4), 311-325.

Tyler, T. R. (2006). Why People Obey the Law. Princeton: Princeton University Press.

Tyler, T. R., Degoey, P., \& Smith, H. (1996). Understanding why the justice of group procedures matters: a test of the psychological dynamics of the groupvalue model. Journal of Personality and Social Psychology, 70 (5), 913930.

Tyler, T. R., \& Folger, R. (1980). Distributional and procedural aspects of satisfaction with citizen-police encounters. Basic and Applied Social Psychology, 1 (4), 281-292. 
Tyler, T. R., \& Blader, S. L. (2003). The group engagement model: procedural justice, social identity, and cooperative behavior. Personality and Social Psychology Review, 7 (4), 349-361.

Van den Bos, K. (1999). What are we talking about when we talk about novoice procedures? On the psychology of the fair outcome effect. Journal of Experimental Social Psychology, 35, 560-577.

Van den Bos, K. (2003). On the subjective quality of social justice: the role of affect as information in the psychology of justice judgments. Journal of Personality and Social Psychology, 85 (3), 482-498.

Van den Bos, K. (2005). What is responsible for the fair process effect? In Greenberg, J. \& Colquitt, J. A. (Eds.), Handbook of Organizational Justice (pp. 273-300). Mahwah, NJ: Lawrence Erlbaum.

Van den Bos, K., Vermunt, R., \& Wilke, H. (1996). The consistency rule and the voice effect: the influence of expectations on procedural fairness judgments and performance. European Journal of Social Psychology, 26 (3), 411-428.

Van den Bos, K., Lind, E. A., Vermunt, R., \& Wilke, H. A. M. (1997). How do I judge my outcome when I do not know the outcome of others? The psychology of the fair process effect. Journal of Personality and Social Psychology, 72, 1034-1046.

Van den Bos, K., Vermunt, R., \& Wilke, H. A. M. (1997). Procedural and distributive justice: what is fair depends more on what comes first than on what comes next. Journal of Personality and Social Psychology, 72 (1), 95104.

Van den Bos, K., Wilke, H. A. M., \& Lind, E. A. (1998). When do we need procedural fairness? The role of trust in authority. Journal of Personality and Social Psychology, 75 (6), 1449-1458. 
Van den Bos, K., Wilke, H. A. M., Lind, E. A., \& Vermunt, R. (1998). Evaluating outcomes by means of the fair process effect: evidence for different processes in fairness and satisfaction judgments. Journal of Personality and Social Psychology, 74 (6), 1493-1503.

Van den Bos, K., Bruins, J., Wilke, H. A. M., \& Dronkert, E. (1999). Sometimes unfair procedures have nice aspects: on the psychology of the fair process effect. Journal of Personality and Social Psychology, 77 (2), 324-336.

Van den Bos, K., Lind, E. A., \& Wilke, H. A. M. (2001). The psychology of procedural and distributive justice viewed from the perspective of fairness heuristic theory. In Cropanzano, R. (Ed.), Justice in the workplace: Volume 2. From theory to practice. Mahwah, NJ: Lawrence Erlbaum.

Van den Bos, K., \& Van Prooijen, J. (2001). Referent cognitions theory: the role of closeness of reference points in the psychology of voice. Journal of Personality and Social Psychology, 81 (4), 616-626.

Van Prooijen, J., Van den Bos, K., \& Wilke, H. A. M. (2002). Procedural justice and status: status salience as antecedent of procedural fairness effects. Journal of Personality and Social Psychology, 83 (6), 1353-1361.

Vermunt, R., \& Steensma, H. (2003). Physiological relaxation: stress reduction through fair treatment. Social Justice Research, 16 (2), 135-149.

Wemmers, J., Van der Leeden, R., \& Steensma, H. (1995). What is procedural justice: criteria used by Dutch victims to assess the fairness of criminal justice procedures. Social Justice Research, 8 (4), 329-350.

Wenzel, M. (2006). A letter from the tax office: compliance effects of informational and interpersonal justice. Social Justice Research, 19 (3), 345364. 